\title{
THE MAKING OF THE CHIEF HEALTH OFFICER'S REPORT, 2000
}

\section{Tim Churches}

Epidemiology and Surveillance Branch

NSW Department of Health

The design and production of a large and eclectic document such as The health of the people of New South Wales: Report of the Chief Health Officer, 2000 presents a number of organisational and technical challenges. This article outlines some of those challenges, and discusses how they were addressed in the making of the report.

The Report of the Chief Health Officer is intended to be an authoritative source of population health indicators for a broad audience, which includes health professionals and health service administrators, other public and private sector service providers, students, and members of the general public. In order to achieve this goal the following design criteria were adopted:

- the report must contain as broad as possible a range of population health status measures and indicators;

- the report should present these indicators in as consistent and coherent a manner as possible, given the diversity of the indicators;

- the report must be understandable by the general public, while containing sufficient detail to satisfy more technically-oriented users;

- the indicators contained in the report need to be as up-to-date as possible;

- an historical context should be provided for each indicator-in other words, time trends should be shown, not just the current value of an indicator;

- comparisons of the values of indicators for NSW with those for other States, Territories and countries should be provided where data is available;

- indicators should be available for geographical and other sub-populations within NSW, in order to maximise the relevance of the report to local communities, and to particular demographic and cultural subgroups;

- the report must be as free of errors as possible;

- mechanisms should be provided which facilitate the reuse and republication of the indicators contained in the report, in order to maximise their exposure and effect.

In order to meet these criteria, the following approaches were taken:

- Wherever possible, indicators were calculated or derived directly from original unit record data (microdata).
- The current and historical unit record data, as well as denominator (population estimate) data, were all drawn from a common source: the HOIST population health data warehouse facility operated by Epidemiology and Surveillance Branch. The HOIST facility employs a range of checking and audit procedures to ensure that the data it contains is as accurate and as up-to-date as possible.

- All data manipulation, and all graph and table production, was performed by a suite of carefully tested software modules which implemented a standardised set of algorithms (details of which appear in the appendices of the report). This purely 'programmatic' approach to the preparation of all quantitative information contained in the report was adopted because it reduces the opportunity for human error, permits auditing and, most importantly, it allows indicators to be easily and efficiently updated as more recent data becomes available. A formal auditing process was used, in which key aspects of all of the computer programs used in the preparation of the report were checked by someone other than the author(s) of each program.

- Each indicator is presented on a separate page. The majority of pages conform to a common layout, comprising a graph (chosen from a small number of basic graph designs, so that the reader develops some familiarity with the graphing paradigms used), a table which repeats the data presented in the graph, a set of footnotes providing details on the data sources and other characteristics of the indicator, a number of paragraphs of explanatory or commentary text, and a set of references and pointers to further information on each indicator.

- Past experience has shown that simple typographical errors, made while transcribing or retyping quantitative data in graphs or tables, were a major source of mistakes that are almost impossible to detect by proofreading. To avoid this, data was produced in formats which avoided any need for transcription or retyping at any stage of the report production process.

- The report is available in both printed and interactive Web (Internet) versions. Initially, these two versions contain the same information. However, the Web version of the report will be updated as more recent source data becomes available. It will also be extended to include both a greater range of indicators and greater levels of detail, such as indicators at the local government area level. 
- Each page of the Web version includes links, which permit the reader to download electronic copies of the graph and table displayed on that page in a form that facilitates re-use in presentations and in other reports or documents. For example, downloaded graphs may be embedded in a word processed document, or downloaded tabular data may be imported into a spreadsheet or graphics package.

However, there is still room for improvement:

- Some of the types of graphs in the current report, particular ones which display statistical confidence limits, may appear unnecessarily complex to some readers. To address this problem, we are developing techniques that will allow the reader to dynamically choose (at least in the Web version of the report) his or her preferred type of graph, such as a traditional vertical bar chart without confidence limits, as opposed to the rather more technically oriented horizontal 'dot' chart with confidence limits which is currently used throughout the report.

- It would be desirable for more comparative or 'benchmarking' data to be included in the report. The limiting factor here is the availability of comparable data from other jurisdictions. This issue is currently being addressed by a number of groups, such as the National Public Health Partnership.

- As greater detail and depth is added to the Web version of the report, the design of the user interface and navigation aids becomes more important to ensure that users can easily find what they are looking for. User testing of different interfaces and navigation aids is required to guide future development.

- Although it is possible to provide more and more detail at relatively low marginal cost, it is impossible to satisfy every user's specific requirements. To address these needs, an interactive facility in which users can specify highly customised or 'bespoke' analyses, graphs or tables is required. Although it is technically feasible to provide access to such a facility via the Internet right now, there are issues of 'disclosure control' (maintenance of confidentiality) and the interpretation of analyses based on small numbers which need to be addressed before such facilities can be made widely available.

\section{NSW PUBLIC HEALTH BULLETIN}

The NSW Public Health Bulletin is a publication of the NSW Department of Health. The editor is Dr Lynne Madden, Manager, Public Health Training and Development Unit, NSW Department of Health.The assistant editor is Ms Allison Salmon, and Dr Michael Giffin is production manager.

The Bulletin aims to provide its readers with population health data and information to motivate effective public health action.

\section{Submission of articles}

Articles, news and comments should be 1000 words or less in length and include a summary of the key points to be made in the first paragraph. References should be set out in the Vancouver style, described in the New England Journal of Medicine, 1997; 336: 309-315. Send submitted articles on paper and in electronic form, either on disc (Word for Windows is preferred), or by email. The article must be accompanied by a letter signed by all authors. Full instructions for authors are available on request from the editor.

\section{Editorial correspondence}

Please address all correspondence and potential contributions to The Editor, NSW Public Health Bulletin, Locked Mail Bag 961, North Sydney NSW 2059 or to Lmadd@doh.health.nsw.gov.au. Tel (02) 93919956 , Fax (02) 93919232.

\section{Distribution}

Please contact your local Public Health Unit or telephone (02) 93919942 to obtain copies of the NSW Public Health Bulletin or to notify us of a change of address. The Bulletin can be accessed via the Internet from the Department's Web site at: www.health.nsw.gov.au/public-health/phb/phb.html.

Back issues can be obtained from the Public Health Training and Development Unit, Locked Mail Bag 961, North Sydney NSW 2059. 